\title{
Enhanced spontaneous Raman scattering and gas composition analysis using a photonic crystal fiber
}

\author{
Michael P. Buric,,$^{1,2}$ Kevin P. Chen, ${ }^{1,2}$ Joel Falk, ${ }^{1,2, *}$ and Steven D. Woodruff ${ }^{1}$ \\ ${ }^{1}$ National Energy Technology Laboratory, 3610 Collins Ferry Road, Morgantown, West Virginia 26507, USA \\ ${ }^{2}$ Department of Electrical and Computer Engineering, University of Pittsburgh, 348 Benedum Hall, \\ Pittsburgh, Pennsylvania 15261, USA \\ *Corresponding author: falk@engr.pitt.edu
}

Received 2 April 2008; revised 9 July 2008; accepted 11 July 2008; posted 15 July 2008 (Doc. ID 94564); published 6 August 2008

\begin{abstract}
Spontaneous gas-phase Raman scattering using a hollow-core photonic bandgap fiber (HC-PBF) for both the gas cell and the Stokes light collector is reported. It was predicted that the HC-PBF configuration would yield several hundred times signal enhancement in Stokes power over a traditional free-space configuration because of increased interaction lengths and large collection angles. Predictions were verified by using nitrogen Stokes signals. The utility of this system was demonstrated by measuring the Raman signals as functions of concentration for major species in natural gas. This allowed photomultiplier-based measurements of natural gas species in relatively short integration times, measurements that were previously difficult with other systems. (C) 2008 Optical Society of America

OCIS codes: $\quad 300.6450,060.5295$.
\end{abstract}

\section{Introduction}

\section{A. Overview}

Spontaneous Raman scattering has long been used to determine the composition of gaseous samples. These gas concentration measurements have been hampered by low Raman cross sections and resultant low signal strengths [1], which have limited the technique's usefulness as a gas-phase sensor. If the signal could be increased, Raman scattering would be ideal for a process stream sensor for hydrocarbon combustion. The work reported in this paper is motivated by the need for real-time measurement of the relative percentages of methane, ethane, and propane that flow along a natural gas pipeline. Detailed knowledge of these gas concentrations is useful in limiting pollutant output and in increasing burning efficiency in large natural-gas-fired turbines. The real-time composition information of natural gas provides continuous heat content and flame speed values for

0003-6935/08/234255-07\$15.00/0

(C) 2008 Optical Society of America feedback control. The requirement to conduct quick composition measurements of low-Ramancross-section gases has motivated our search for enhanced Raman signal collection techniques.

In this paper we demonstrate that spontaneous Raman output power in a hollow-core, photonic bandgap fiber (HC-PBF) is enhanced by several hundred times over that obtained using a traditional, $90^{\circ}$ free-space collection system [2]. In our sensing system, pump light travels through an approximately meter-long HC-PBF that is filled with an analyte gas. The Stokes radiation generated within the entire HC-PBF propagates down the length of fiber, and the exiting light is imaged onto the input slit of a grating spectrometer. The long interaction length in the HC-PBF serves to enhance the Raman signatures of gas species over those available from conventional Raman systems. It is noted that extremely low-loss $(<1.2 \mathrm{db} / \mathrm{Km}) \mathrm{HC}-\mathrm{PBF}$ s have been recently reported [3]. These should lead to much longer usable lengths of HC-PBF and to larger enhancements, limited only by stimulated scattering [4]. The utility of the HC-PBF system reported here was demonstrated by measuring the Raman signals 
as functions of concentration for major species in natural gas.

\section{B. Related Work, Capillary Tubes}

It is noted that classical hollow capillary tubes have also been used for the enhancement of nonlinear optical interactions [5-13]. These capillaries work best with rays that propagate at grazing incidence, i.e., when only the fundamental $\mathrm{EH}_{11}$ or low-order waveguide mode propagates [14-18]. High-order, nongrazing modes have high loss and thus are of limited use for enhancement of nonlinear interactions. Most classic hollow-capillary, nonlinear optics enhancement experiments that have been reported describe output signals that required phase matching and consequently did not benefit from the collection of a large number of waveguide modes or the collection of a large solid-angular output [5-9,11,12]. Thus these experiments are not strongly affected by the requirement of grazing incidence. There have, however, been reported enhancements of spontaneous Raman signals using hollow-core capillary tubes for collinear propagation of pump and Stokes signals $[10,13]$. The Stokes signals generated therein depend linearly on the number of modes that propagate to the output of the tube with low loss. Enhancements reported by Schwab and McCreery [13] were 30-50 times a free-space configuration and presumably were limited by the significant propagation losses that occurred except for rays propagating at grazing incidence to the walls of the tube. These losses limited the number of modes (and hence the solidangular output) that could be collected.

\section{Related Work in HC-PBFs}

Recent related work has used HC-PBFs to take advantage of the long interaction lengths available when both a laser beam and a gas are confined to the small cross section of the hollow-core fiber. In particular, optical sources based on stimulated rotational Raman scattering, and sensitive absorption measurements in HC-PBFs have been reported [4,19-22]. Stimulated Raman scattering in HC-PBFs depends on the enhanced gain possible in the fiber's confined volume. The stimulated Raman exponential gain coefficient depends on $(P / A) L$ (pump power $P$ per unit area $A$ times interaction length $L$ ) and is enhanced in the HC-PBF system by the small pump beam crosssectional area and long fiber length [23]. Absorption measurement sensitivities are also increased by the long lengths possible when a HC-PBF absorption cell is employed [24]. The signal enhancement realized when a HC-P $\overline{B F}$ is used to contain the Raman medium and to collect the spontaneous Raman emission is of a somewhat different character.

In Section 2 of this paper we theoretically estimate the enhancement factor of Raman scattering in HCPBFs over the conventional Raman configuration. The theoretical estimation is validated with experimental results using atmospheric $\mathrm{N}_{2}$ gas (Subsection 3.A). The application of the HC-PBF Raman sensing system to the measurement of natural gas composition is described in Subsection 3.B. The significance of this work and conclusions are summarized in Section $\underline{4}$.

\section{Theory-Enhancement of Spontaneous Raman Scattering in HC-PBFs: Numerical Calculations for HC- 580-1 Fiber}

In general, spontaneous Stokes output power $P_{s}$ is found from

$$
P_{s}=\sigma D P_{L} \Omega_{0}
$$

where $\sigma$ is the Raman scattering cross section, $D$ is the density of the sample, $P_{L}$ is the incident laser power, $L$ is the Raman interaction length, and $\Omega_{0}$ is the solid-angular Stokes output that is detected [2]. Thus comparisons of the efficiency of Raman detection systems are in large part a comparison of the $L \Omega_{0}$ products of competing systems.

In the classic, free-space, $90^{\circ}$ gas Raman configuration using a grating spectrometer, the pump laser beam is directed parallel to the input slit of the spectrometer. The beam is focused through the analyte gas such that the beam has a depth of field longer than height $H$ of the spectrometer's entrance slit. The pump beam (and resultant Stokes scattering) is imaged onto the entrance plane of the spectrometer. The interaction length $L$ is determined by height $H$ of the spectrometer slit, and the value of $\Omega_{0}$ is determined by the $f$-number $(f / \#)$ of the spectrometer. If the imaging magnification is unity, $\Omega_{0}=$ $\pi\left(1 /(2 f \#)^{2}\right)$ is the solid-angular acceptance, and the collected Stokes power in this configuration is given by $[\underline{25}, \underline{26}]$

$$
P_{s}=\sigma D H \pi\left(1 /(2 f \#)^{2}\right) P_{L} .
$$

The magnification of any collection optics $(M>1)$ decreases the effective value of $L$ by $M$ and increases the effective value of $\Omega_{0}$ by $M^{2}$. The magnification therefore increases the Raman signal by $M$. The maximum value of $M$ is severely restricted by the requirement that the magnified Raman signal fit through the narrow dimension of the spectrometer's slit and is typically of order unity [25].

For Raman scattering inside a hollow-core singletransverse-mode HC-PBF-based system, Stokes radiation is generated and transmitted down the length of the fiber inside the gas-filled hollow core. If the optical loss of the fiber is very low, the collected Stokes power will obey Eq. (1), where $L=L_{f}$ is the length of the fiber and $\Omega_{0}$ is the solid angle accepted by the fiber.

Guiding in the hollow-core HC-PBF is forced by a photonic bandgap within the cladding material. This bandgap extends through a sizable region of the spectrum, $100 \mathrm{~nm}$ for the HC-580-1 HC-PBF used in this work [27]. The photonic bandgap prevents propagation into the cladding in this wavelength region. This 
particular HC-PBF supports a single transverse waveguide mode over this low-loss spectral range.

The Stokes radiation produced and collected inside the HC-PBF is proportional to the number of Raman radiation modes collected, which in turn is proportional to the Stokes solid-angle $\Omega_{0}$ collected by the fiber [26]. This solid-angle acceptance can be estimated in three similar ways. In the first method, used in the numerical estimates given in Section 3 of this paper, the solid-angular acceptance is calculated from the measured numerical aperture of the fiber, defined as $\sin \theta$, where $\theta$ is the angular acceptance of the fiber. For small $\theta, \Omega_{0} \approx \pi \theta^{2}$. The numerical aperture of the fiber used herein is 0.12 and $\Omega_{0} \approx 0.045 \mathrm{sr}$ [27]. The second estimate of $\Omega_{0}$ is derived by observing the near-Gaussian nature of the transverse profile of the propagating mode. The $1 / e^{2}$ intensity angular divergence of a Gaussian mode is estimated as $\theta \approx \lambda /\left(\pi w_{0}\right)$, where $w_{0}$ is the Gaussian beam radius. For small $\theta, \Omega_{0} \approx \pi \theta^{2} \approx \lambda^{2} /\left(\pi w_{0}^{2}\right)$ [26]. For the HCPBF investigated in this paper $w_{0} \approx 2.1 \sqrt{2}=$ $2.96 \mu \mathrm{m}[27]$ and $\Omega_{0} \approx 0.0124 \mathrm{sr}$ at the nitrogen Stokes wavelength ( $584.5 \mathrm{~nm}$ for a $514.5 \mathrm{~nm}$ pump laser) reported in Section 3 of this paper. We note that, since this angular measurement excludes any ray whose intensity is smaller than $1 / e^{2}$ of peak intensity, it is not surprising that this estimate leads to a lower value for $\Omega_{0}$ than one based on the HC-PBF's numerical aperture. We also note that if we estimate the area of the Gaussian mode $A \approx \pi w_{0}{ }^{2}$ we can write $A \Omega_{0} \approx \lambda^{2}$. The Gaussian mode estimate is similar in reasoning to the third estimate based on a blackbody mode analysis. The number of Raman radiation modes can be calculated by a standard mode count, identical to the mode count used in theoretical treatments of blackbody radiation [26]. That estimate, based on quantization of modes in a finite space, leads to the conclusion that each Raman mode occupies a solid angle $\Omega \approx \lambda^{2} / A$, where $\lambda$ is the Stokes wavelength and $A$ is the cross-sectional area of the quantizing propagation medium [26]. For the single spatial mode observed to propagate in the $\mathrm{HC}-\mathrm{PBF}$, this means that the solid-angular collection is given by $\Omega_{0} \approx$ $\lambda^{2} / A$. Taking $A$ as the area of the HC-580-1 HCPBF core (diameter $4.9 \mu \mathrm{m}), \Omega_{0} \approx 0.048 \mathrm{sr} \quad(\lambda=$ $514.5 \mathrm{~nm})$. The three estimates for the solid-angular acceptance of the HC-PBF differ by only a factor of roughly 3.6 from smallest to largest. In the numerical analysis given in this paper we used the value of $\Omega_{0}$ based on the first estimate, which is the only estimate derived from an experimental measurement of the fiber's angular collection properties. We note that the HC-PBF's angular acceptance estimates are similar in magnitude to that imposed by the angular collection of the $f \#=6.4$ spectrometer used in the experiments reported below. For free-space Raman collection with $\Omega_{0}$ determined entirely by the spectrometer $(M=1), \Omega_{0}=\pi\left(1 /(2 f \#)^{2}\right)=0.019 \mathrm{sr}$.

Equations (1) and (2) show that the ratio of Stokes power collected by the PBF to that collected in the free-space configuration is determined by the ratio of the solid-angle-interaction length products for the two configurations. Because similar acceptance angles are available from both free-space and HCPBF Raman systems, the total Stokes power collected is largely dependent on the Raman interaction length $\mathrm{L}$. The advantage of the HC-PBF or any hollow-core fiber is that the solid-angle light collection is integrated over the length of the fiber and reduced to a near point emission at the end of the fiber for transmission to a spectrometer. This length is typically much longer in the HC-PBF system $\left(L=L_{f}\right)$ than in the free-space system $(L=H)$. This point is discussed in more detail in Section $\underline{3}$.

\section{Experiments}

\section{A. Atmospheric Pressure Nitrogen}

Predicted enhancements of the HC-PBF system were demonstrated by using atmospheric-pressure nitrogen in both the HC-PBF and the $90^{\circ}$ classic free-space configurations (Fig. 1). In the HC-PBF system, an

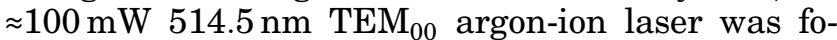
cused into a $1.5 \mathrm{~m}$ length of Crystal Fibre, HC-58001 hollow-core photonic bandgap fiber via an $\sim 10 \mathrm{~mm}$ effective focal length (EFL) aspheric singlet and a three-axis Thorlabs fiber-launch stage. The HC580 fiber has loss $<1 \mathrm{~dB} / \mathrm{m}$ at wavelengths between 510 and $590 \mathrm{~nm}$ and $<1.5 \mathrm{~dB} / \mathrm{m}$ at wavelengths between 590 and $610 \mathrm{~nm}$ [27]. The fiber supports a single transverse, near-Gaussian waveguide mode at these wavelengths and has a numerical aperture of 0.12 . Greater than $60 \%$ input coupling efficiency was measured. At the output end of the HC-PBF, a collimator was used to limit the optical divergence of the output beam prior to its passing through a Semrock, holographic edge filter $[28,29]$. After passing the filter, the remaining Stokes radiation was focused into a $0.55 \mathrm{~m}$ focal length grating spectrometer, and the spectrometer's output was measured with an EMI 9789A photomultiplier tube operated in a photon counting mode. The quantum efficiency of the detection system was not optimized.

In the free-space configuration, the pump beam was reflected to travel parallel to the spectrometer's entrance slit and was focused to an $\sim 150 \mu \mathrm{m}$ radius beam waist in front of the slit with a $0.5 \mathrm{~m}$ EFL lens. The pump beam propagated $16 \mathrm{~cm}$ away from the entrance slit. A $2.54 \mathrm{~cm}$ diameter, $4 \mathrm{~cm}$ EFL lens

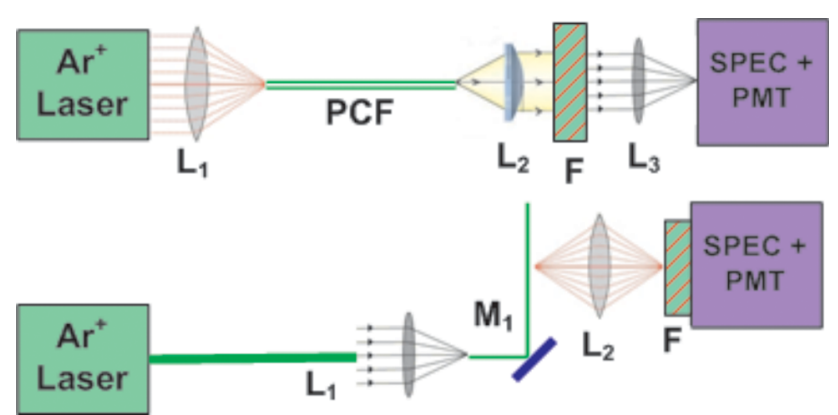

Fig. 1. (Color online) Experimental gas Raman systems. 
provided $M=1$ imaging of the Stokes radiation onto the slit. The Semrock edge filter was placed directly in front of the slit to reduce the scattered pump light entering the spectrometer. The spectrometer's (Horiba $\mathrm{J}-\mathrm{Y}$ iHR550) dispersion is $1.37 \mathrm{~mm} / \mathrm{mm}$.

Figure 2 shows the two resultant spectra collected from the $\overline{\mathrm{H} C}-\mathrm{PBF}$ based system and traditional $90^{\circ}$ scattering system. The free-space spectrum was taken with a $0.5 \mathrm{~mm}$ wide, $10 \mathrm{~mm}$ high input slit. This wide slit was used only to facilitate alignment. The HC-PBF Raman spectrum was taken with $0.2 \mathrm{~mm}$ wide, $10 \mathrm{~mm}$ high slits, and exhibits a higher resolution accordingly. Either slit width is wide enough to pass the entire nitrogen or oxygen spectral line. Linewidths in both experiments were instrument limited. The detection system in both experiments was identical. Figure 2 shows nitrogen and oxygen spectral lines imposed on a background that increases with shorter wavelengths. The figure shows that the $\mathrm{N}_{2}$ Raman line at $2331 \mathrm{~cm}^{-1}(584.5 \mathrm{~nm})$ collected from a $1.5 \mathrm{~m}$ piece of air-filled HC-PBF is more than 130 times greater than that obtained in free space. This enhancement is consistent with that predicted
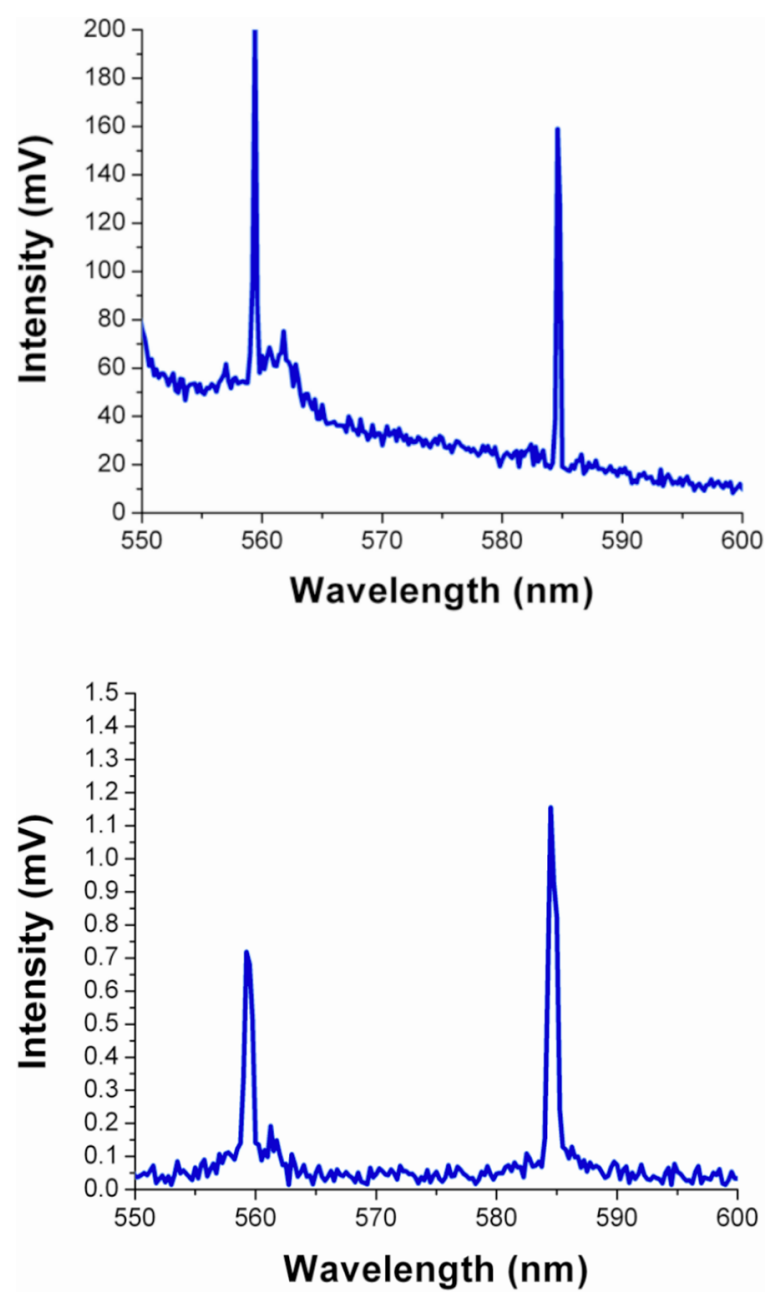

Fig. 2. (Color online) Raman spectra from (top) the HC-PBF system and (bottom) the free-space system . A signal intensity of $1 \mathrm{~V}$ corresponds to $10^{5}$ count/s. The peaks near 560 and $585 \mathrm{~nm}$ are due to $\mathrm{O}_{2}$ and $\mathrm{N}_{2}$ Raman scattering. from Eqs. (1) and (2), which show that the ratio $R$ of powers detected is $R=L_{f} \Omega_{f} P_{l f} / H \pi\left(1 /(2 f \#)^{2} P_{l o}\right.$, where $L_{f}$ and $\Omega_{f}$ are the fiber length and the solid angle of the Stokes signal collected by the fiber. $P_{l o}=$ $160 \mathrm{~mW}$ and $P_{l f}=45 \mathrm{~mW}$ are the laser powers used in the free-space and fiber experiments, respectively. For our fiber $\left(L_{f}=1.5 \mathrm{~m}, \Omega_{f} \approx 0.045 \mathrm{sr}\right)$ the predicted enhancement ratio $R \approx 100$. The measured enhancement (Fig. 2) is $152 \mathrm{mV} / 1.15 \mathrm{mV}=132$. Raman Stokes signals are proportional to the pump power, and this measured enhancement implies an expected enhancement of $132 \times(160 / 45)=470$ for equal pump powers. (We note that all Raman signal levels reported in the text of this paper are corrected for background signals; i.e., the background, primarily due to Raman scattering from the silica present in the cladding, is subtracted from the signal levels shown in the figures.)

The Stokes power $\left(P_{s}\right)$ measured in the free-space experiment is consistent with that expected from the known Raman cross section for nitrogen [25]. That power, calculated from Eq. (2) $\left(\sigma D=\overline{5} \times 10^{-12}\right.$ $\left.(\mathrm{cm} \mathrm{sr})^{-1}, \quad M=1, \quad \Omega_{0}=0.019 \mathrm{sr}\right) \quad$ is $\quad P_{s}=6.7 \times$ $10^{-15} \mathrm{~W}$. The free-space Raman signal shown in Fig. 2 (peak value $1.15 \mathrm{mV}$ ) corresponds to 115 photoelectrons/s. The measured quantum efficiency of the phototube at $\lambda=585 \mathrm{~nm}$ is $1.28 \%$, and the spectrometer's transmission is $44 \%$, which implies a generated Raman power of $7.1 \times 10^{-15} \mathrm{~W}$, in good agreement with the theoretical prediction. Also visible in both spectra is the oxygen Stokes line at $1556 \mathrm{~cm}^{-1}(559 \mathrm{~nm})$. If we account for the difference in Raman cross section between oxygen and nitrogen [25] and the difference in Stokes wavelength $\lambda_{s}$ (the Stokes photon number varies as $\lambda_{s}{ }^{-3}$ ), the ratio of nitrogen/oxygen Stokes signals (proportional to Stokes photon numbers) should be 2.7. The displayed ratio of photon numbers for the free-space experiment is 1.6-1.7. (This ratio in the HC-PBF experiment is not the same because of observed chromatic aberrations in the optical train. These aberrations become noticeable only when the spectrometer slits are small.) The quantum efficiency of the photomultiplier tube at $559 \mathrm{~nm}$ (oxygen Stokes wavelength) is a factor of 1.6 higher than at $585 \mathrm{~nm}$ (nitrogen Stokes wavelength), accounting for most of the difference between the predicted and the displayed ratio.

In the HC-PBF spectrum, a strong amorphous silica Raman band is present. The silica signal is several times greater than the gas Raman signals and peaks near the pump wavelength. The silica Raman signal is present throughout the HC-PBF transmission band and is indicative of a small portion of the laser light propagating in the silica portion of the fiber's cladding. We have experimentally observed that this noise can be reduced by spatial filtering at the fiber's output, i.e., by minimizing the collection of light that leaks outside the fiber's core. 


\section{B. Natural Gases}

The utility of our HC-PBF Raman system was demonstrated by the detection and measurement of the components of natural gas (methane, ethane, and propane). A schematic diagram of the HC-PBF Raman system used is shown in Fig. 3. High-purity natural gas components were introducéd into a stainless steel chamber designed to contain the gases at high pressure. The gases were fed through a $1 \mu \mathrm{m}$ sintered stainless steel filter and a subsequent $0.1 \mu \mathrm{m}$ polypropylene filter before entering the HC-PBF. These filters were used to prevent contamination of the fiber cladding and core holes during measurement.

The same pump laser and input coupling experimental arrangement was utilized as in previously described atmospheric nitrogen measurement experiments. In this case, the light input end of the HC-PBF also served as the vent for excess gases escaping the fiber. The other end of the HC-PBF was butt coupled to a length of $110 \mu \mathrm{m}$ core diameter, multimode fiber using an etched silica $\mathrm{V}$ groove and UVcuring epoxy. The butt-coupling allowed for collection of the output pump and Raman light while maintaining an air gap between the two fibers of a few micrometers. The entire coupling assembly was placed inside the high-pressure chamber with the $\mathrm{HC}$ PBF and multimode fibers exiting the chamber through two feedthroughs. The gap between the two fibers allowed analyte gases to pass into the HC-PBF core and out into the atmosphere at the other end of the fiber. At the output end of the large multimode fiber, the same collimation, filtering, and measurement apparatus was employed as in the atmospheric nitrogen experiment. The total measured flow through the $\mathrm{HC}-\mathrm{PBF}$ core and cladding was used to calculate the flow rate in the fiber core, assuming uniform laminar flow in the core and cladding regions $[30,31]$. Measured flow rates predict a fill time of $6 \mathrm{~s}$ for a $25 \mathrm{~cm}$ fiber, and thus filling of the entire fiber core can occur quickly enough to achieve a gas concentration reading in a few seconds. Because relatively large signal-to-noise ratios are achieved in this system, the limiting factor in measurement time is the time required to fill the fiber, and not the time required to integrate and time average the signal, as would be the case in a free-space configuration.

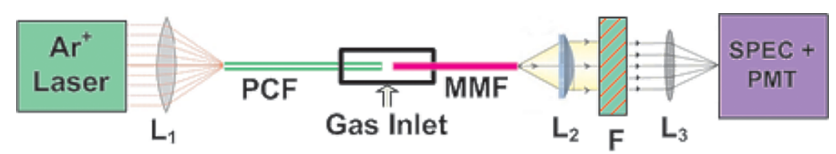

Fig. 3. (Color online) HC-PBF Raman experimental system. A $514.5 \mathrm{~nm} \mathrm{Ar}^{+}$laser beam, $<1 \mathrm{~W} \mathrm{TEM}_{00}$, is focused by a $10 \mathrm{~mm}$ EFL aspheric singlet $\left(\mathrm{L}_{1}\right)$ into an HC-580-01 HC-PBF. Inside a high-pressure gas chamber, the HC-PBF is butt coupled to a $110 \mu \mathrm{m}$ core multimode fiber (MMF). The output from the multimode fiber is collimated (fiber collimator $\mathrm{L}_{2}$ ), and argon laser light is removed by using holographic edge filter $\mathrm{F}$. Lens $\mathrm{L}_{3}$ (focal length $60 \mathrm{~nm}$ ) delivers the Stokes signal to a JY IHR550 Spectrometer (SPEC) and an EMI 9789A phototube (PMT).
The two most relevant portions of the resultant natural gas Raman spectra are shown in Fig. 4 . In the first portion, from 535 to $545 \mathrm{~nm}$, distinct lines are observed in ethane and propane. In the second portion, from 600 to $610 \mathrm{~nm}$, emissions from methane, ethane, and propane are simultaneously observed.

After all relevant hydrocarbon lines had been located and evaluated, the concentrations of each gas were varied while observing the intensity of relevant Raman lines. To accomplish this, a single gas was injected into the fiber at 100 psi (6.9 bar). The resultant Raman line intensity of interest was measured, and the gas was diluted with high-purity nitrogen until the natural gas Raman line was no longer visible. An examination of the individual
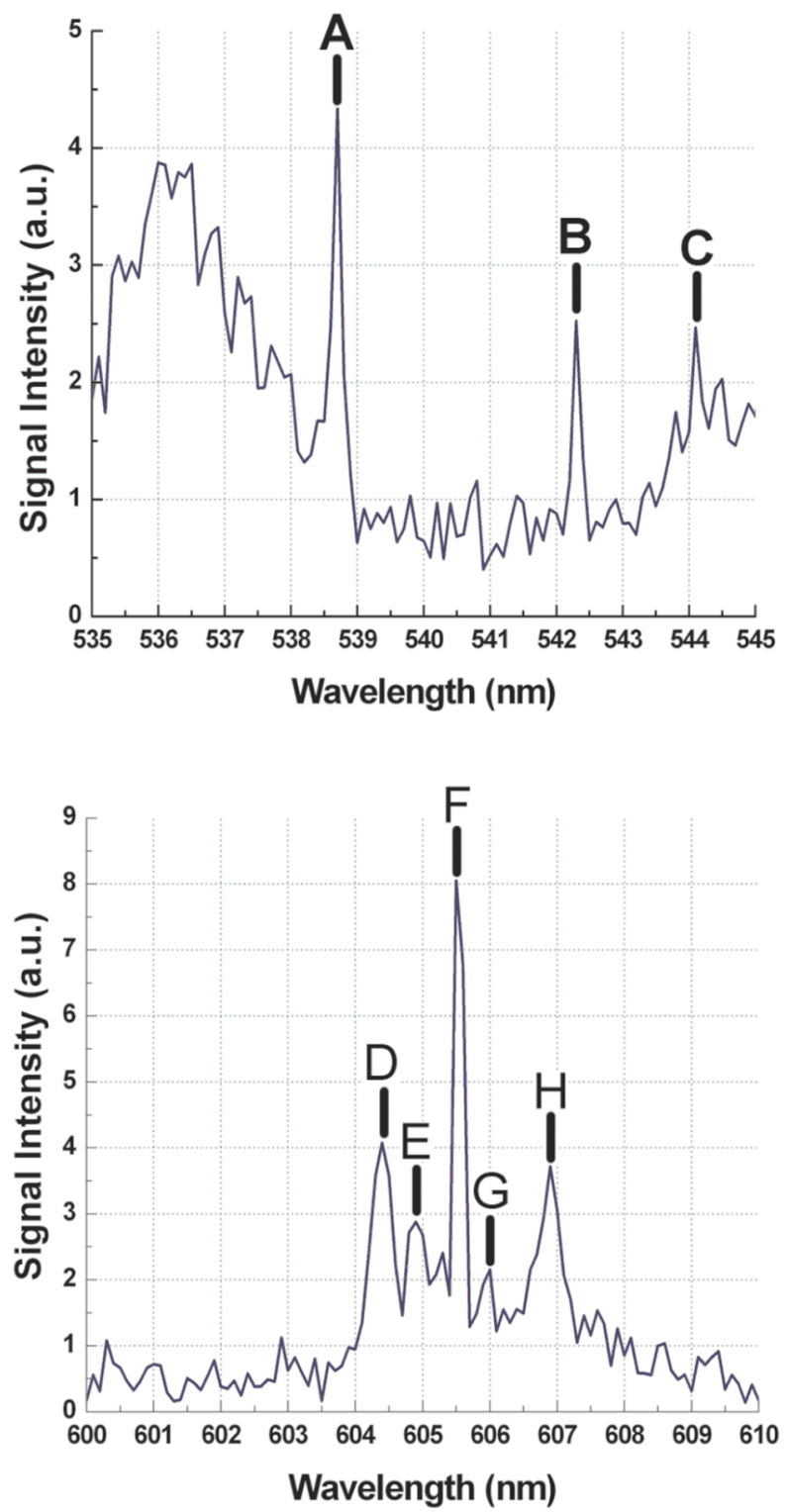

Fig. 4. (Color online) Raman spectra of natural gas: top, $535-545 \mathrm{~nm}$; bottom, $600-610 \mathrm{~nm}$. Identification of indicated spectral lines: A, $870 \mathrm{~cm}^{-1}$ propane; $\mathrm{B}, 1000 \mathrm{~cm}^{-1}$ ethane; $\mathrm{C}, 1060 \mathrm{~cm}^{-1}$ propane; D, $2883 \mathrm{~cm}^{-1}$ propane; $\mathrm{E}, 2895 \mathrm{~cm}^{-1}$ ethane; $\mathrm{F}, 2917 \mathrm{~cm}^{-1}$ methane; G, $2937 \mathrm{~cm}^{-1}$ propane; $\mathrm{H}, 2995 \mathrm{~cm}^{-1}$ ethane. 
and mixed hydrocarbon spectra at varying pressures did not show any deviation from the spectral shapes shown in Fig. 4. Figure 5 shows the intensity of two such Raman lines (methane $2917 \mathrm{~cm}^{-1}$ and ethane $2955 \mathrm{~cm}^{-1}$ ) versus gas concentration. The straight lines are least-squares linear fits to the experimental data. Each data point shown in Fig. 5 is an average of 51 measurements of the Stokes Raman signal taken at $1 \mathrm{~s}$. The error bars shown in the figure are \pm one standard deviation of the 51 data points. The ethane data show a near-linear variation of the Raman intensity with constituent concentration. The variation of the methane data from linear is substantial. We speculate that the nonlinear variation in methane data is caused by the flow of high-pressure gases, which causes movement in the coupling between the HC-PBF and the multimode fiber used to deliver the Raman signal to the spectrometer. The ethane data were taken first, at which time the coupling was stable.

In addition to the methane, ethane, and propane Raman signatures, a stronger continuous amorphous silica Raman band was seen starting near the $514.5 \mathrm{~nm}$ pump wavelength, peaking at about $525 \mathrm{~nm}$, and decreasing through the remainder of the photonic bandgap fiber's transmission band. The peak of the silica Raman band at $525 \mathrm{~nm}$ (not shown in Fig. 4) is approximately 7 times greater than the silica Raman local maximum at $536 \mathrm{~nm}$. The solid silica Raman signal is consistent with previous observations [32] and is accentuated by the long pump-beam-silica-fiber interaction length in the multimode collection fiber. This silica Raman signal provides the noise limit in the present system, and future experimental work designed for an optimum signal-to-noise ratio should use an all hollowcore fiber system to minimize silica Raman noise [33]. In the nonoptimized system illustrated here, the lower detection limit for methane, for example, was only about $0.5 \%$ owing to the absence of background subtraction, optimized photon counting, stray light elimination, and limited detector quan-

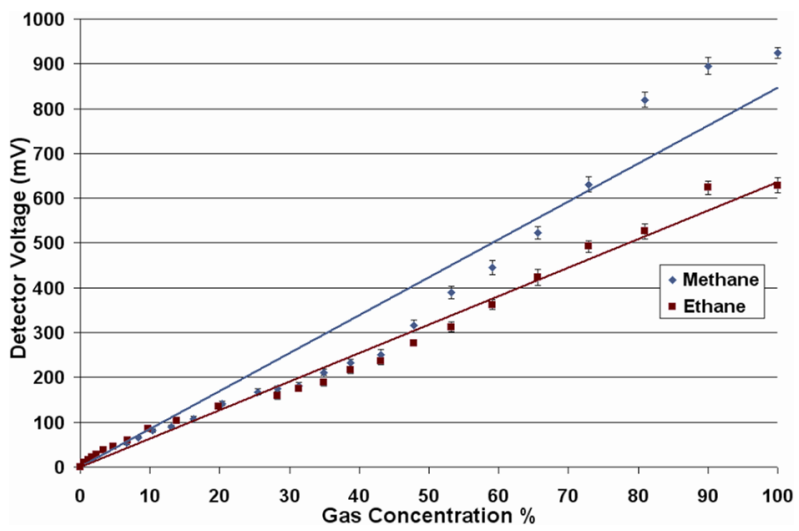

Fig. 5. (Color online) Measured Raman intensities of methane $\left(2917 \mathrm{~cm}^{-1}\right)$ and ethane $\left(2995 \mathrm{~cm}^{-1}\right)$ Raman lines with varying gas concentration. The straight lines are linear fits to the experimental points anchored at the zero intercept. tum efficiency. Were these parameters optimized, we infer that using the same HC-PBF system would result in detection limits $\sim 300$ times lower than that realized with a free-space configuration.

\section{Significance and Conclusions}

A hollow-core photonic bandgap fiber was used both as a Raman gas cell and as the means for collection of spontaneously emitted Stokes light. This method produced enhanced spontaneous Raman signals that allowed gas concentration measurements that were several hundred times more sensitive than comparable free-space methods, and the method was significantly better than comparable capillary tube systems. Analysis of the intensity of Stokes signals produced by this system yielded signals in good agreement with those measured. Because similar solid angles may be collected for either the free-space or HC-PBF configuration, a comparison between the systems is essentially a comparison of usable Raman interaction lengths. Long, commercial, hollow-core, photonic bandgap fibers with losses $<0.4 \mathrm{~dB} / \mathrm{m}$ are available, and thus fibers as long as 10-20 m should be useful for Raman signal enhancement [33]. This implies that signal enhancements of $L_{f} / \overline{H \geq} 1000$ [Eqs. (1) and (2)] should be achievable.

We have successfully applied the HC-PBF Raman system to real-time measurement of molecular constituents of natural gas. These experiments provide a crucial step toward the realization of a cost-effective, all-fiber natural gas monitoring system, as well as providing a basis for the understanding of Raman interactions inside photonic bandgap fibers.

This technical effort was performed in support of the National Energy Technology Laboratory's research in Energy Systems and Dynamics under RDS contract DE-AC26-04NT41817 in addition to support from National Science Foundation (NSF) grant 0639234.

\section{References}

1. D. A. Long, Raman Spectroscopy (McGraw-Hill, 1977).

2. R. L. McCreery, Raman Spectroscopy for Chemical Analysis (Wiley-Interscience, 1977).

3. P. Roberts, F. Couny, H. Sabert, B. Mangan, D. Williams, L. Farr, M. Mason, A. Tomlinson, T. Birks, J. Knight, and P. St. J. Russell, "Ultimate low loss of hollow-core photonic crystal fibres," Opt. Express 13, 236-244 (2005).

4. P. Russell, "Photonic crystal fibers: a historical account," IEEE LEOS Newsletter 21(5), 11-15 (October 2007).

5. A. M. Zheltikov, "Ultrashort light pulses in hollow waveguides,” Phys. Usp. 45, 687-718 (2002).

6. S. Sensarn, S. N. Goda, G. Y. Yin, and S. E. Harris, "Molecular modulation in a hollow fiber," Opt. Lett. 31, 2836-2838 (2006).

7. M. Nisoli, S. De Silvestri, and O. Svelto, "Generation of high energy $10 \mathrm{fs}$ pulses by a new pulse compression technique" Appl. Phys. Lett. 68, 2793-2795 (1996).

8. R. B. Miles, G. Laufer, and G. C. Bjorklund, "Coherent antistokes Raman scattering in a hollow dielectric waveguide," Appl. Phys. Lett. 30, 417-419 (1977).

9. A. M. Burzo, A. V. Chugreev, and A. V. Sokolov, "Optimized control of generation of a few cycle pulses by molecular modulation," Opt. Commun. 264, 454-462 (2006). 
10. S. M. Howdle, K. Stanley, V. K. Popov, and V. N. Bagratashvili, "Can high-pressure Raman spectroscopy be simplified? A microscale optical-fiber capillary cell for the study of supercritical fluids," Appl. Spectrosc., 48, 214-218 (1994).

11. S. O. Konorov, A. B. Fedotov, A. M. Zheltikov, and R. B. Miles, "Phase-matched four-wave mixing and sensing of water molecules by coherent anti-stokes Raman scattering in largecore-area hollow photonic-crystal fibers," J. Opt. Soc. Am. B 22, 2049-2053 (2005).

12. A. B. Fedotov, S. O. Konorov, V. P. Mitrokhin, E. E. Serebryannikov, and A. M. Zheltikov, "Coherent antiStokes Raman scattering in isolated air-guided modes of a hollow-core photonic-crystal fiber," Phys. Rev. A 70, 045802 (2004).

13. S. D. Schwab and R. L. McCreery, "Remote, long-pathlength cell for high-sensitivity Raman spectroscopy," Appl. Spectrosc., 41, 126-130 , (1987).

14. M. J. Renn and R. Rastel, "Particle manipulation and surface patterning by laser guidance," J. Vac. Sci. Technol. B 16, 38593863 (1998).

15. R. Paster, A. Sturthers, R. Ringle, J. Rogers, C. Rohde, and P. Geiser, "Laser trapping of microscopic particles for undergraduate experiments," Am. J. Phys. 68, 993-1001 (2000).

16. A. Yariv and P. Yeh, Optical Waves in Crystals (WileyInterscience, 1984).

17. C. C. Gregory, "Attenuation, modal, and polarization properties of $n<1$, hollow dielectric waveguides," Appl. Opt. 32, 5302-5309 (1993).

18. E. A. J. Marcatili and R. A. Schmeltzer, "Hollow metal and dielectric waveguides for long distance optical transmission and lasers," Bell Syst. Tech. J. 43, 1783-1809 (1964).

19. P. Russell, "Photonic crystal fibers," Science 299, 358-362 (2003).

20. F. Benabid, G. Antonopoulos, J. C. Knight, and P. St. J. Russell, "Stokes amplification regimes in quasi-cw pumped hydrogenfilled hollow-core photonic crystal fiber," Phys. Rev. Lett. 95, 213903 (2005).
21. T. Ritari, J. Tuominen, H. Ludvigsen, J. C. Petersen, T. Sorensen, T. P. Hansen, and H. R. Simonsen, "Gas sensing using air-guiding photonic bandgap fibers," Opt. Express 12, 4080-4087 (2004).

22. P. Russell, "Photonic crystal fiber: finding the holey grail," Opt. Photon. News, 18(7), 26-31 (July/August 2007).

23. F. Benabid, J. C. Knight, G. Antonopoulos, and P. St. J. Russell, "Stimulated Raman scattering in a hydrogen filled hollow-core photonic crystal fiber," Science 298, 399-402 (2002).

24. S.-G. Li, S.-Y. Liu, Z.-Y. Song, Y. Han, T.-L. Cheng, G.-Y. Zhou, and L.-T. Hou, "Study of the sensitivity of gas sensing by use of index-guiding photonic crystal fibers," Appl. Opt. 46, 51835188 (2007).

25. W. Fenner, H. A. Hyatt, J. M. Kellam, and S. P. S. Porto, "Raman cross section of some simple gases," J. Opt. Soc. Am. 63, 73-77 (1973).

26. A. Yariv, Quantum Electronics, 3rd ed. (Wiley, 1989).

27. HC-580 HC-PBF datasheet, retrieved 1 June 2007 from http:// www.crystal-fibre.com/datasheets/HC-580-01.pdf.

28. M. M. Carrabba, K. M. Spencer, C. Rich, and D. Rauh, “The utilization of a holographic Bragg diffraction filter for Rayleigh line rejection in Raman spectroscopy," Appl. Spectrosc. 44, 1558-1561 (1990).

29. "RazorEdge long wave pass Raman filter," http://www. semrock.com/Catalog/RamanEdgeFilter_spectra.htm

30. J. B. Evett and C. Liu, Fundamentals of Fluid Mechanics (McGraw-Hill, 1987).

31. J. Henningsen and J. Hald, "Dynamics of gas flow in hollow core photonic bandgap fibers," Appl. Opt. 47, 2790-2797 (2008).

32. B. E. A. Saleh and M. C. Teich, Fundamentals of Photonics, 2nd ed. (Wiley-Interscience, 2007).

33. S. O. Konorov, C. J. Addison, H. G. Schulze, R. F. B. Turner, and M. W. Blades, "Hollow-core photonic crystal fiber-optic probes for Raman spectroscopy,” Opt. Lett. 31, 1911-1913 (2006). 